\title{
Synchronous Endometrial and Ovarian Cancer With Sigmoid Colon Metastasis One Year After Primary Surgery: A Case Report
}

\author{
Kadir Guzin ${ }^{1}$, Burcin Karamustafaoglu Balci ${ }^{*}$, Kemal Sandal ${ }^{1}$, Ahmet Gocmen ${ }^{1}$, Ozgur Ekinci², \\ Abdullah Aydin ${ }^{3}$, Meryem Yuvruk ${ }^{3}$
}

\begin{abstract}
Introduction: We describe a patient who had ovarian and endometrial cancer which metastasized to sigmoid colon one year after surgery.

Case Presentation: A 53-year-old woman was admitted with the complaints of abdominal pain, abdominal distension and postmenopausal bleeding. Her transvaginal ultrasound scan revealed a cystic mass containing papillary projections with the dimensions of $6.5 \times 5 \times 4 \mathrm{~cm}$ on the right ovary. Level of (carbohydrate antigen) (CA) 125 was 223 IU. Dilation and curettage revealed endometrioid adenocarcinoma. Debulking surgerywas carried out. Histopathological diagnosis was grade 2 adenocarcinoma with squamous differentiation for endometrial cancer and grade 2 endometrioid adenocarcinoma with squamous and mucinous differentiation for ovarian cancer. The stage was $1 \mathrm{~A}$ for endometrial cancer and $1 \mathrm{~A}$ for ovarian cancer. 12 months after the operation CA 125 level was 112 IU. Positron emission tomography (PET) scan showed a small lesion $(1.5 \times 1.5 \mathrm{~cm})$ in the pelvic cavity with increased fluorodeoxyglucose (FDG) uptake. Five months after the chemotherapy, CA 125 level elevated from 10 to 60 IU and subsequent magnetic resonance imaging (MRI) revealed a tumoral mass with the dimensions of $3 \times 2.2 \mathrm{~cm}$. A second laparotomy was performed and the metastasis was excited. The tumor was endometrioid adenocarcinoma with infiltration in the serosa and muscularis propria of sigmoid colon.

Conclusion: It is needed to consider the possibility of double cancer in the diagnosis and treatment of gynecological malignancies. Keywords: Endometrial neoplasms, Neoplasm metastasis, Ovarian neoplasms, CA-125 antigen
\end{abstract}

\section{Introduction}

The presence of synchronous primary malignancies in the female genital tract is relatively rare. Primary tumors in two organs within a single person is called synchronous primary malignancies. The two types of synchronous cancers commonly reported have been ovarian cancer and endometrial cancer $(1,2)$. Synchronous primary tumor of the endometrium and ovary occur in up to $10 \%$ of all ovarian cancer cases and $5 \%$ of all endometrial cancer cases (3). Patients with simultaneous uterine and ovarian cancers do generally show a better prognosis than those with metastatic disease. (2,4-6). We describe herein, a patient whose disease was not consistent with this data, she had ovarian cancer (stage 1A) and endometrial cancer (stage 1A) which metastasized to sigmoid colon one year after surgery.

\section{Case Presentation}

A 53-year-old postmenopausal women, with a history of 5 pregnancies, 2 deliveries, 1 dilatation and curettage and 2 missed abortus, admitted to our clinic with the complaints of abdominal pain, abdominal distension and postmenopausal bleeding. Her family and previous disease histories were unremarkable. Her physical examination and transvaginal ultrasound scan revealed free fluid in the abdominal cavity and a cystic mass containing papillary projections with the dimensions of $6.5 \times 5 \times 4 \mathrm{~cm}$ on the right ovary. Tumor marker (carbohydrate antigen) (CA) 125 was elevated, $223 \mathrm{IU}$. The dilatation and curettage was performed and the histopathological diagnosis of endometrial curettage was endometrioid adenocarcinoma. Computed tomography revealed no metastasis. Under the diagnosis of endometrial cancer and probably ovarian cancer; malignant ascites sampling, radical hysterectomy, bilateral salpingo-oophorectomy, pelvic and paraaortic lymphadenectomy, omentectomy, appendectomy and cytologic sampling of the undersurface of the diaphragm were carried out (tumor free). Histopathological diagnosis of endometrial cancer was grade 2 adenocarcinoma with squamous differentiation (stage 1A) with less than

Received 9 March 2015, Accepted 19 August 2015, Available online 1 October 2015

${ }^{1}$ Department of Obstetrics and Gynecology, Goztepe Training and Research Hospital, Medeniyet University, Istanbul, Turkey ${ }^{2}$ Department of General Surgery, Goztepe Training and Research Hospital, Medeniyet University, Istanbul, Turkey. ${ }^{3}$ Department of Pathology, Goztepe Training and Research Hospital, Medeniyet University, Istanbul, Turkey.

*Corresponding author: Burcin Karamustafaoglu Balci, Department of Obstetrics and Gynecology, Goztepe Training and Research Hospital, Medeniyet University, Istanbul, Turkey. Email: burcinkaramustafaoglu@yahoo.com 
half myometrial invasion (myometrial thickness $17 \mathrm{~mm}$, depth of invasion $6 \mathrm{~mm}$ ) (Figure 1A). Histopathological diagnosis of ovarian tumor was grade 2 endometrioid adenocarcinoma with squamous and mucinous differentiation (Figure 1B). The capsule of the ovarian tumor was intact, pelvic and paraaortic lymph nodes were negative. The stage was $1 \mathrm{~A}$ for endometrial cancer and $1 \mathrm{~A}$ for ovarian cancer. Therefore, no adjuvant chemotherapy was planned. After the operation CA 125 was within normal limits (10 IU). The patient was on follow-up and 12 months after the operation CA 125 level was 112 IU. PET scan showed a small lesion $(1.5 \times 1.5 \mathrm{~cm})$ in the pelvic cavity with increased fluorodeoxyglucose (FDG) uptake. She was treated with 9 weekly courses of paclitaxel and carboplatin. The CA 125 level after the chemotherapy was 10 IU. Five months after the chemotherapy CA 125 level elevated to $60 \mathrm{IU}$ and subsequent magnetic resonance imaging (MRI) revealed a tumoral mass with the dimensions of $3 \times 2.2 \mathrm{~cm}$ in abdominal cavity, above psoas muscles, between intestinal loops. A second laparotomy was performed and a $2.5 \times 3 \mathrm{~cm}$ in size tumor on sigmoid colon was seen. The tumor was placed on the serosal side of the colon and was not causing bowel obstruction. No tumoral lesion was seen on psoas muscles. Multiple biopsies were taken. They were all negative. The metastasis was excited from $5 \mathrm{~cm}$ below the lower border and from $10 \mathrm{~cm}$ up the upper border of the tumor and end-to-end anastomosis was done. The examination of the tumoral mass that was extracted showed that there was endometrioid adenocarcinoma with infiltration in the serosa and muscularis propria of sigmoid colon (Figure 1C). Immunohisto- chemically, tumor cells were positive for pax8 (Figure 1D) and negative with WT1 and ER. The margins were clear (negative margins). The patient is still on follow up and no recurrence has occured.

\section{Discussion}

When there is contemporarily endometrial and ovarian malignancies, the differential diagnosis between metastasis and synchronous primary tumors is difficult.

Histopathologic workup differentiating synchronous carcinoma from metastatic carcinoma was done. We found that each tumor was situated away from each other and that each tumor was not a metastasis of the other. Uterine cancer had less than half myometrial invasion and the grade of both uterine and ovarian tumors were almost the same. In such cases, the diagnosis is synchronous primary tumors, not metastasis. Consequently, the patient was diagnosed as having double simultaneous tumors. Histopathological diagnosis of the mass that was extracted from the sigmoid colon was endometrioid carcinoma and the specimen showed positive reaction to pax 8 which is one of markers in distinguishing genital tract carcinomas. This positivity showed that the tumor in sigmoid colon was an endometrioid carcinoma metastasis. However, it could not be exactly decided whether the malignant cells in this mass were derived from endometrial and/or ovarian cancers. The WT1 is a marker for serous carcinoma; the tumor in sigmoid colon was negative for WT1; which shows that it is not serous carcinoma of the endometrium and/or ovary.

The coexistence of synchronous multiple primary malig-
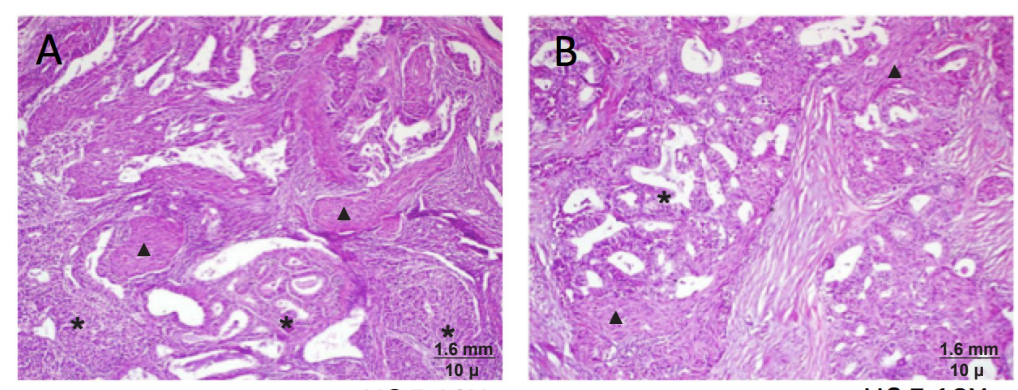

H\&E $10 X$

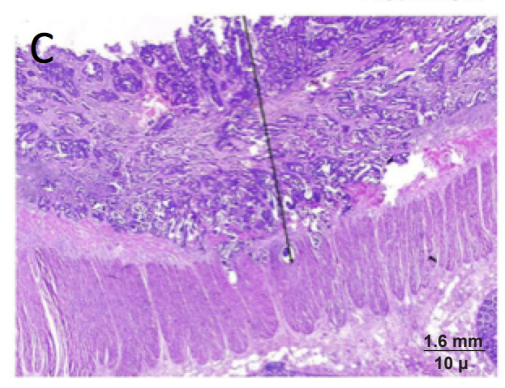

H\&E $2 X$

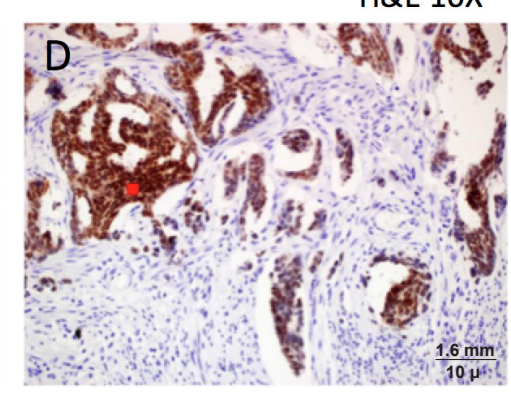

PAK8 2X

Figure 1. (A) Endometrium: Endometrioid adenocarcinoma grade 2. Atypical glandular formation of endometrioid adenocarcinoma with squamous metaplasia (H\&E 10X). $\mathbf{\Delta}$ : Squamous differentiation area. *: Endometrioid carcinoma. (B) Ovarian tumor: Endometrioid adenocarcinoma in the right ovary. Endometrioid adenocarcinoma with atypical glands is seen (H\&E 10X). $\mathbf{\Delta}$ : Squamous differentiation area. *: Endometrioid carcinoma. (C) Endometrioid adenocarcinoma in serosa of the sigmoid colon. Atypical glands are invading the outer part of the muscularis propria of the colon (H\&E 2X). Arrow: Endometrioid carcinoma on the serosal surface and muscularis propria of the sigmoid colon. (D) Nuclear Pax-8 positivity in the tumor cells of the metastasis in the colon. This marker shows that the tumor is originating from genital tract (pax-8 20X). m: Pax8 positivity in the nuclei of tumor cells. 
nancies is very interesting. A case of synchronous primary carcinomas in five different organs of a female genital tract has been reported (7). Even though the treatment is similar as if they were single primary tumors, the prognosis of patients with synchronous primary cancers are generally better than the patients with metastatic disease of a single tumor $(2,3)$. In a prospective GOG study, patients with simultaneous uterine and ovarian carcinomas showed a 5 -year survival of $86 \%$ and a 10 -year survival of $80 \%$ and their prognosis was better than those with metastatic disease (3). A possible explanation may be that generally ovarian tumors are diagnosed at advanced stages $(75 \%-85 \%$ stage III/IV), while the majority of ovarian cancers in patients with synchronous multiple primary neoplasms are diagnosed at an earlier stage (8). The rationale underlying this fact is probably the symptoms that the other primary tumor cause. Another explanation may be the fact that although ovarian and endometrial cancer are generally diagnosed in the sixth or seventh decade (8), it has been demonstrated that the median age of the patients with synchronous primary tumors ranged from 41 to 54 years, suggesting that multiple primaries tend to occur at least $10-20$ years earlier in life $(2,9,10)$, which indicates a positive prognostic criterion. Meanwhile, our patient's prognosis was not as good as expected; she experienced a metastasis only one year after surgery. Increasing CA 125 level indicates and supports the new onset of tumor on sigmoid colon.

We aimed to share our experience with a patient who had two synchronous cancers in genital tract which were concurrent endometrial and ovarian cancers. It is necessary to consider the presence of double cancer in the diagnosis and treatment of gynecological cancers.

\section{Ethical Issues}

Not applicable.

\section{Conflict of Interests}

The authors declare no conflict of interests.

\section{Acknowledgements}

The authors declare that there is no acknowledgement.

\section{References}

1. Ayhan A, Yalcin OT, Tuncer ZS, Gurgan T, Kucukali
T. Synchronous primary malignancies of the female genital tract. Eur J Obstet Gynecol Reprod Biol. 1992;45(1):63-66.

2. Eifel P, Hendrickson M, Ross J, Ballon S, Martinez A, Kempson R. Simultaneous presentation of carcinoma involving the ovary and the uterine corpus. Cancer 1982;50(1):163-170.

3. Zaino R, Whitney C, Brady M, Degeest K, Burger R, Buller R. Simultaneously detected endometrial and ovarian carcinomas - a prospective clinicopathologic study of 74 cases: a Gynecologic Oncology Group Study. Gynecol Oncol. 2001;83(2):355-362.

4. Caldarella A, Crocetti E, Taddei GL, Paci E. Coexisting endometrial and ovarian carcinomas: a retrospective clinicopathological study. Pathol Res Pract. 2008;204(9):643-8. doi: 10.1016/j.prp.2008.02.001.

5. Chiang YC, Chen CA, Huang CY, Hseieh CY, Cheng WF. Synchronous primary cancers of the endometrium and ovary. Int J Gynecol Cancer. 2008;18(1):159-64.

6. Pearl ML, Johnston CM, Frank TS, Roberts JA. Synchronous dual primary ovarian and endometrial carcinomas. Int J Gynaecol Obstet. 1993;43(3):305312.

7. Atasever M, Yilmaz B, Dilek G, Akcay EY, Kelekci S. Synchronous primary carcinoma in 5 different organs of a female genital tract: an unusual case and review of the literature. Int J Gynecol Cancer. 2009;19(4):802807. doi: 10.1111/IGC.0b013e3181a39b95.

8. Barakat RR, Grigsby PW, Sabbatini P, Zaino RJ. Corpus: Epithelial Tumors. In: Hoskins W, Perez C, Young R, eds. Principles and Practice of Gynecologic Oncology. Philadelphia, USA: Lippincott; 2000:919960.

9. Soliman PT, Slomovitz BM, Broaddus RR, et al. Synchronous primary cancers of the endometrium and ovary: a single institution review of 84 cases. Gynecol Oncol. 2004;94(2):456-62.

10. Uccella S, Cha SS, Melton LJ 3rd, et al. Risk factors for developing multiple malignancies in patients with endometrial cancer. Int J Gynecol Cancer. 2011;21(5):896-901. doi: 10.1097/ IGC.0b013e318219711f.

Copyright $\odot 2015$ The Author(s); This is an open-access article distributed under the terms of the Creative Commons Attribution License (http://creativecommons.org/licenses/by/4.0), which permits unrestricted use, distribution, and reproduction in any medium, provided the original work is properly cited. 